\title{
Saturation estimates and chromatic adaptation
}

GERALD H. JACOBS

DEPARTMENT OF PSYCHOLOGY AND DEFENSE RESEARCH LABORATORY, THE UNIVERSITY OF TEXAS

Category estimates of spectral saturation were obtained from three observers under neutral adaptation and under three conditions of chromatic adaptation. The data so obtained show that chromatic adaptation causes a shift in the location of minimal spectral saturation toward the spectral locus of the adapting light. The existence of secondary minima and enhancement effects in spectral saturation are also noted.

Chromatic adaptation produces pronounced alterations in spectral appearance, the most obvious change being a shift in perceived hue. The characteristics of this type of change have been described indirectly in a number of studies using matching techniques and directly by both descriptive reports and colornaming procedures. In addition to shifts in hue, chromatic adaptation also produces important changes in spectral saturation, but these changes have apparently not been assessed in any experimental manner.

It is only recently, in fact, that attempts to measure spectral saturation directly have appeared. The earlier approaches to saturation, as to hue and brightness, were through the use of matching tasks but there are some obvious reasons why matching has probably not been used to evaluate spectral saturation under chromatic adaptation. Most important of these is the fact that under chromatic adaptation changes occur not only in spectral saturation but also in both hue and brightness. Thus, in order to match completely, it would be necessary to allow the observer to adjust wavelength and intensity, as well as colorimetric purity, and the specification of the resulting change would be complex. In addition, information about spectral saturation, as about other characteristics of spectral appearance, can be obtained from matching results only by inference. Apparently some sort of a more direct approach is needed. Recent studies of saturation have employed scaling procedures in an attempt to investigate the nature of the scale relating colorimetric purity and spectral saturation, typically by means of magnitude estimation (Onley et al, 1963; Panek \& Stevens, 1966; Indow \& Stevens, 1966). Using that approach it would be possible to derive scale functions for all spectral wavelengths under a number of different adaptive states. Since that would be a tedious approach for a large number of wavelengths and several adaptive conditions, in the present experiment a category estimation procedure was utilized in an attempt to provide some information about spectral saturation under chromatic adaptation.

\section{METHOD}

The apparatus used in this experiment has been described previously (Jacobs \& Gaylord, in press). In brief, the stimulus beams of the optical system originated from two sources: a tungsten filament lamp was the source for the adaptation beam, while the test beam originated from a grating monochromator which provided a stimulus having a spectral bandpass of $15 \mathrm{~nm}$. These two beams were brought from right angles to a common focus at an electromagnetic shutter and from there followed the same path to a final lens where they were presented to the eye in Maxwellian view of $40^{\circ}$. The position of the shutter controlled which of the beams passed on through the optical system: with the shutter in one position, the observer saw the adaptation beam; with the shutter in the other position the adaptation beam was blocked and the test beam was directed onto the final lens. The shutter position was controlled automatically through a timing circuit which regulated durations of the adaptation and test lights.

Spectral stimuli from 440 to $650 \mathrm{~nm}$, taken at $10 \mathrm{~nm}$ steps, were used. The adaptation conditions used were: "neutral," for which only neutral density filters were inserted into the tungsten beam, and three conditions of chromatic adaptation obtained by interposing Wratten Filters Nos. 92, 98, and 74 into the tungsten beam (those filters have dominant wavelengths of 636,452 , and $532 \mathrm{~nm}$, respectively). The luminances of the adaptation and test lights were all equated at $66 \mathrm{~mL}$ prior to the experiment so that shifts from adaptation to test lights were always between stimuli of equal luminance.

Observers scaled saturation by estimating the chromatic content in a given stimulus light on an 11-point scale where a judgment "0" indicated a complete lack of any chromatic content in the stimulus and a "10" meant a complete lack of any achromatic component, with the intermediate categories used for the majority of cases where both chromatic and achromatic components could be discerned. In practice, of course, the " 0 " end of the scale can be easily defined for the observer, but the upper end of the scale can be defined only through experience with spectral stimuli under the particular conditions of the experiment. Thus the observers in this experiment were given an opportunity to view all the stimuli under neutral adaptation a number of times prior to any data collection, and they were subsequently familiarized with the stimuli under each of the chromatic-adaptation conditions.

Only one adaptation condition was examined in a single experimental session; the session started with a 5-min. adaptation to the appropriate light. Following initial adaptation, test stimuli of $300 \mathrm{msec}$. 

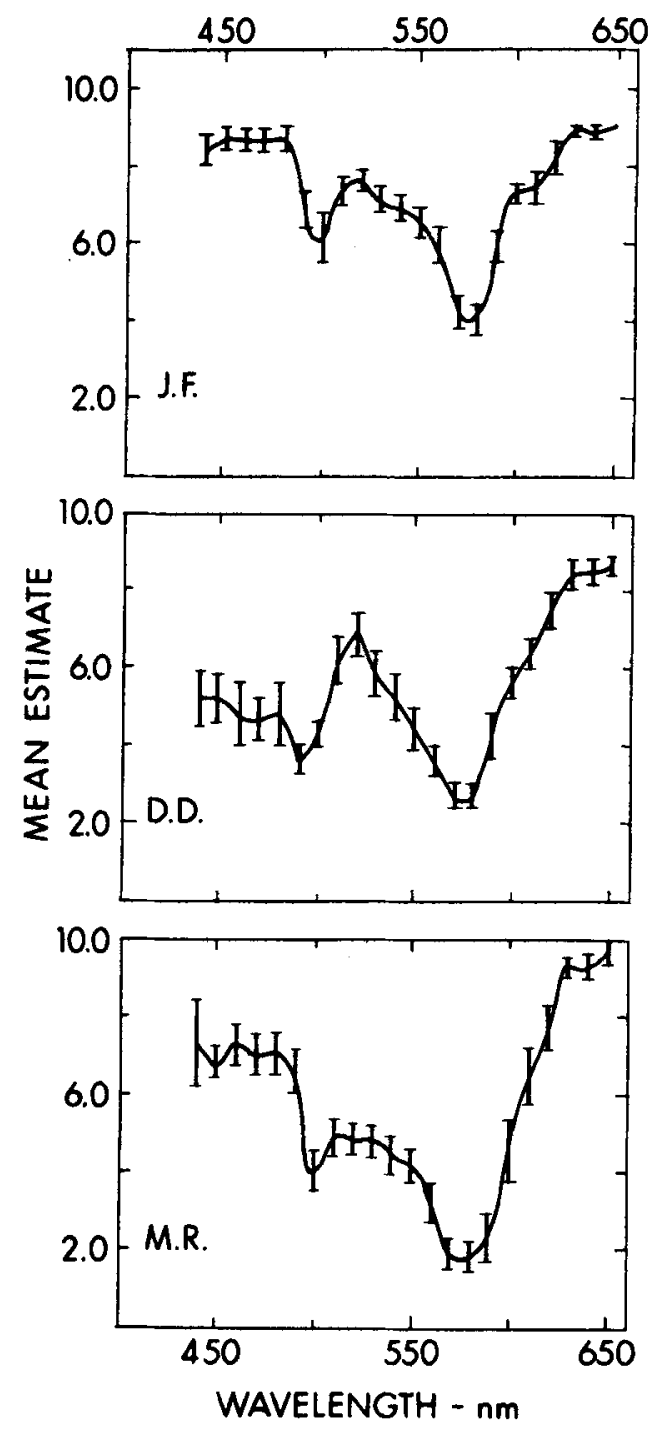

Fig. 1. Mean estimates of saturation for neutral adaptation. The vertical bars enclose one standard deviation.

duration were automatically presented in randomorder once every 18 sec. Between stimuli the relevant adaptation condition prevailed. Each daily session consisted of four presentations each of 22 test wavelengths. Data collection continued until 30 judgments had been recorded for every test wavelength under each of the four adaptation conditions.

Three males, ages 16, 21, and 22, were the observers; two of them served under all four adaptation conditions while the third was run under three adaptation conditions. All had normal color vision.

\section{RESULTS AND DISCUSSION}

There is currently considerable debate as to the appropriate status of direct estimation experiments like this one. On the one hand, some have argued (Graham \& Ratoosh, 1962) that estimates produced by an observer cannot be treated like numerical data gathered in more traditional fashion. They propose that the correct manuever in such experiments is to determine that value of the independent variable necessary to generate each of the numerical Judgments and that tactic has been employed in at least one category estimation experiment (Lewls, 1965). On the other hand, Stevens (1966) has listed a large number of direct estimation experiments that yielded data in agreement with those found by other techniques and argues persuasively as to the utility of such experiments.

Regardless of the logical status of such experiments, there are some obvious limitations as to the ways in which obtained estimates can be treated and evaluated. For example, there is no justification for assuming that a certain distance along, say, a mean estimate dimension signifies the same thing under different adaptive states. Thus, quantitative comparisons across conditions are not possible. Nevertheless, it is still
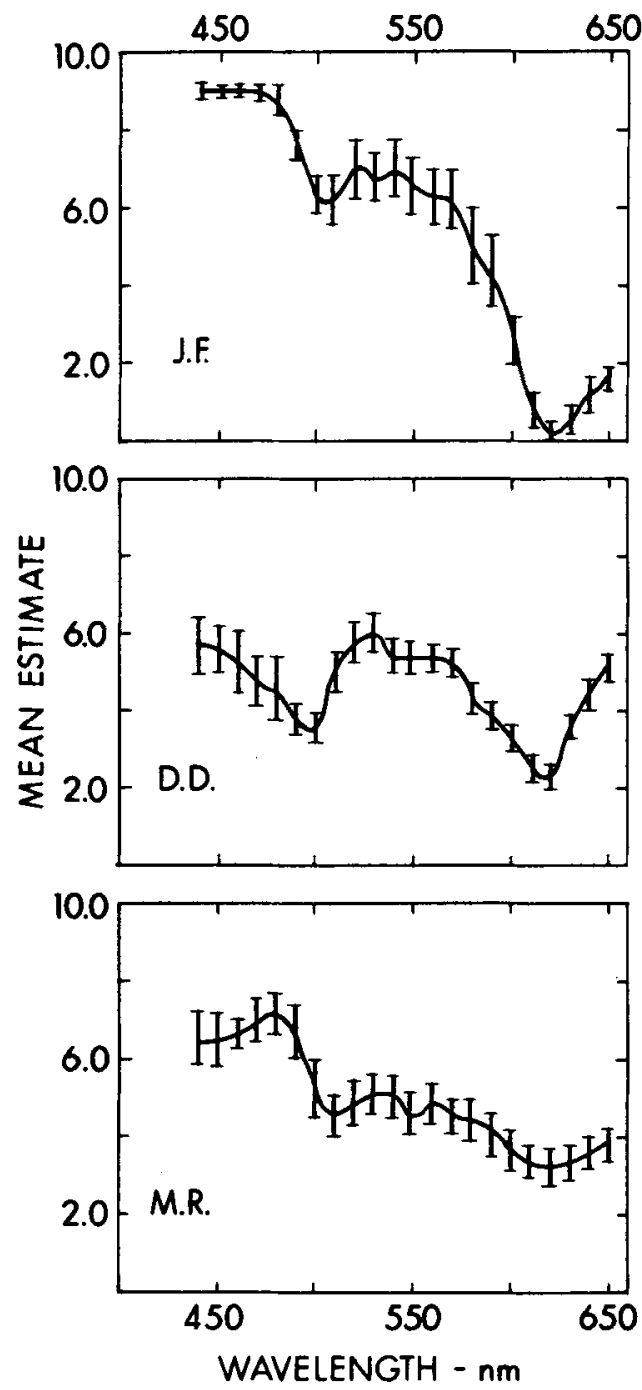

Fig. 2. Mean estimates of saturation for red (dominant wavelength $=636 \mathrm{~nm}$ ) adaptation. 

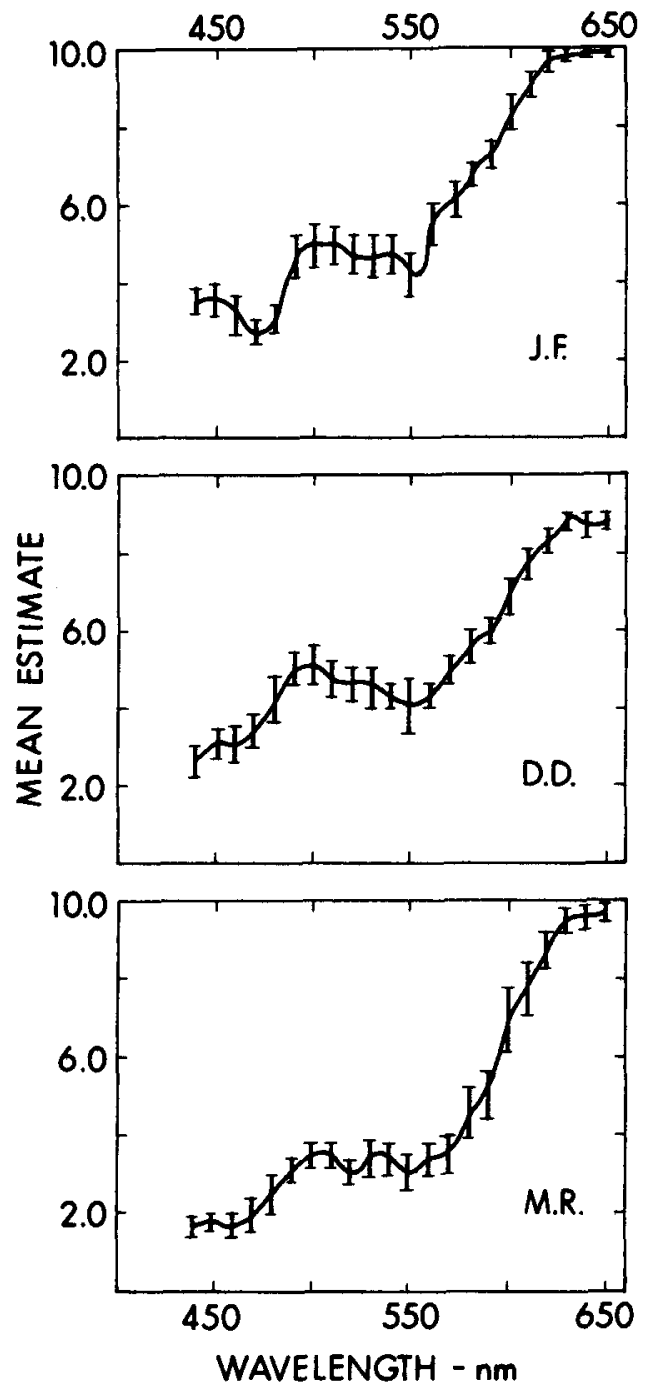

Fig. 3. Mean estimates of saturation for blue (dominant wavelength $=452 \mathrm{~nm}$ ) adaptation.

possible to obtain some qualitative indices as to the change in relative spectral saturation from these data. To that end, mean saturation estimates were calculated for each of the observers under various adaptation conditions. Those functions are depicted in Figs. 1-4. An index of the variability in estimation is also given there for each condition.

A number of features of these functions bear mention. It is, not surprisingly, a relatively difficult task for an observer to judge saturation for a series of heterochromatic stimull. Nevertheless, following initial training the observers of this study were able to perform the task with reasonable consistency as can be seen from the deviation measures given for each condition. Referring first to the neutral-adaptation condition shown in Fig. 1, there are obvious similarities between the functions for all three observers. Although there is considerable variability in the range used by different observers there is clear agreement in the location of minimal estimatesthat is, those points of lowest spectral saturation. The major minimum is at about $580 \mathrm{~nm}$ for all three observers and, in addition, there is also a clearly apparent secondary minimum at 490-500 nm. Saturation increases at both ends of the spectrum with a secondary maximum at about $520 \mathrm{~nm}$ under neutral adaptation.

That chromatic adaptation profoundly alters spectral saturation is clearly evident from the estimation functions of Figs. 2-4. Again, although there is considerable variability between observers, some features are common to all these functions. In general, the point of minimal saturation, seen at about $580 \mathrm{~nm}$ under neutral adaptation, appears under chromatic adaptation near the spectral locus of the adapting light. Thus the minimum moves from $580 \mathrm{~nm}$ under neutral adaptation to $620 \mathrm{~nm}$ under W92 adaptation, to $460 \mathrm{~nm}$ under W98 adaptation, and to a range of 500-550 nm under W74 adaptation. There is also apparent, usually clearly, a secondary region of minimal saturation for each of the chromatic-adaptation conditions. The secondary minimum appears at 490$510 \mathrm{~nm}$ for $W 92$ adaptation, at $550 \mathrm{~nm}$ under W98 adaptation, at $500 \mathrm{~nm}$ under W74 adaptation for one observer and at $570 \mathrm{~nm}$ for the other observer under that same adaptation.

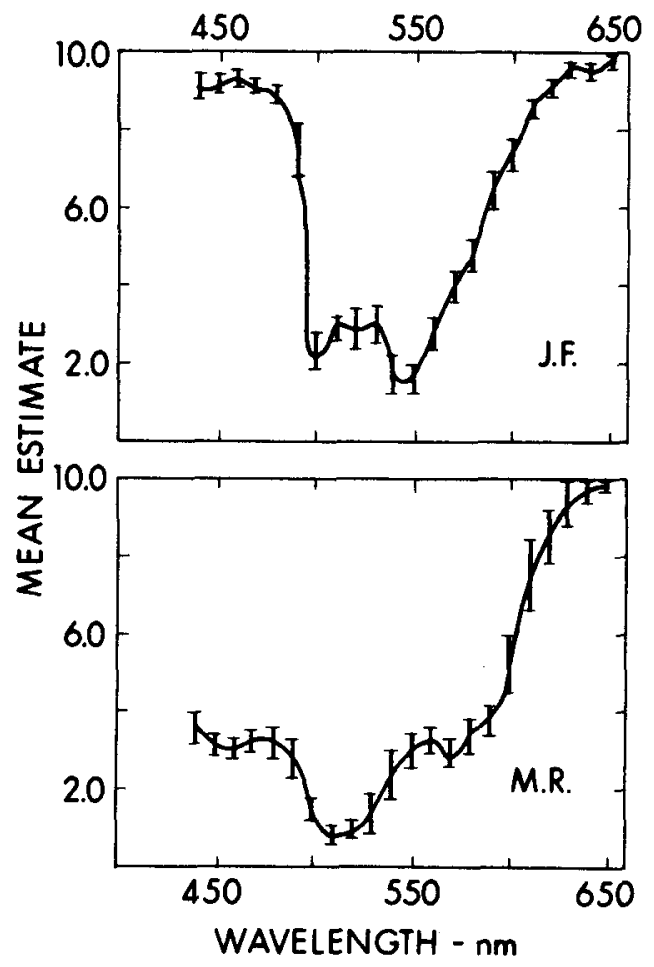

Fig. 4. Mean estimates of saturation for green (dominant wavelength $=532 \mathrm{~nm}$ ) adaptation. 
In addition to changes in the locations of saturation minima described above, chromatic adaptation also produces alterations in the spectral locations of the more saturated stimuli. Although there is no obvious way to quantify it, there is in general an increase in perceived saturation for those spectral regions located approximately complementary to the adapting light. This effect, more obvious in some observers and conditions than in others, is nearly always visible if the functions obtained under chromatic adaptation are compared to the neutral functions for the same observer. These enhancements attest amply to the common observation that it is possible to perceive more highly saturated colors under chromatic adaptation than under conditions of neutral adaptation.

Thore are several features of the present experiment which might be expected to affect perceived saturation which ought to be specifically emphasized. First, stimulus viewing time was brief and controlled in this experiment; viewing time has typically been longer and/or uncontrolled in matching or discrimination studies of spectral saturation. Secondly, the neutral adaptation light was a tungsten source having a color temperature of about $2850^{\circ} \mathrm{K}$ so, specifically, that condition involved adaptation to a slightly yellowish light. Finally, in order to minimize surround induction effects, the retinal region stimulated was large $\left(40^{\circ}\right)$; it has been known for some time that spectral saturation is somewhat dependent on the size of the retinal image (Stevens, 1934).

Given these restrictions, what relationships are there between the estimates of spectral saturation obtained here and those obtained by other approaches utilized previously? There have been a considerable number of attempts to depict relative spectral saturation, using several different procedures (Graham, 1965). These various studies all show minimal spectral saturation at about 570-580 $\mathrm{nm}$ and the direct estimates reported here agree well with that figure. The other prominent dip, seen at about $500 \mathrm{~nm}$ for the observers in this experiment, has not appeared clearly in all previous work although there are hints of such a minimum in some of those data. In particular, the measurements of spectral saturation made by Jameson \& Hurvich (1955) using a cancellation technique also shows evidence for a secondary minimum in the neighborhood of $500 \mathrm{~nm}$. In sum, the functions for noutral adaptation are enough like the functions obtained by other techniques that there appears to be a reasonable validity in the category-estimation procecture utillized here.

As mentioned above, there apparently have been no direct experimental measurements of spectral saturation under chromatic adaptation. The results obtained here, however, seem to be in line with expectation: Chromatic adaptation has generally been said to cause a loss of saturation for that part of the spectrum from which the adapting light is taken and a relative enhancement of saturation for that spectral region complementary to the adapting light (Jameson \& Hurvich, 1956). Finally, there is a rather striking correspondence between the estimation functions reported here and some theoretical functions relating adaptation and spectral saturation derived by Jameson and Hurvich (1956) in the context of their opponent-colors theory. The functions depicted in Fig. 8 of that publication, showing how spectral saturation varies under white, blue, green, and red adaptation, bear close resemblance to the functions of Figs. 1-4 of this study. The measurements described here seem to provide at least qualitative support for that theoretical prediction.

\section{References}

Graham, C. H. Discriminations that depend on wavelength. In C. $\mathrm{H}$. Graham (Ed.), Vision and visual perception. New York: Wiley, 1965. Pp. 350-369.

Graham, C. H., Ratoosh, P. Notes on some interrelations of sensory psychology, perception, and behavior. In S. Koch (Ed.), Psychology: a study of a science. New York: McGraw-Hill, 1962, Vol. IV. Pp. 483-514.

Indow, T., \& Stevens, S. S. Scaling of saturation and hue. Percept \& Psychophys., 1966, 1, 253-271.

Jacobs, G. H., \& Gajlord, H. A. Effects of chromatic adaptation on color naming. Vis. Res., (in press).

Jameson, D., \& Hurvích, C. M. Some quantitative aspects of an opponent-colors theory. I. Chromatic responses and spectral saturation. $J$. Opt. Soc. Amer., 1955, 45, 546-552.

Jameson, D., \& Hurvich, L. M. Some quantitative aspects of an opponent-colors theory, III. Changes in brightness, saturation, and hue with chromatic adaptation. J. Opt. Soc. Amer., 1956. $46,405-415$.

Lewis, M. F. Category judgments as functions of flash luminance and duration. J. Opt. Soc. Amer., 1965, 55, 1655-1660.

Onley, J. W., Klingberg, C. L., Dainotf, M. J., \& Rollman, G. B. Quantitative estimates of saturation. J. Opt. Soc. Amer., 1963 $53,487-493$.

Panek, D. W., \& Stevens, S. S. Saturation of red: A prothetic continuum. Percept. \& Psychophys., 1966, 1, 59-66.

Stevens, $\mathbf{S}$. $\mathbf{S}$. The relation of saturation to the size of the retinal image. Amer. J. Psychol, 1934, 46, 70-69.

Stevens, S. S. Duration, luminance, and the brightness exponent. Percept. \& Psychophys., 1966, 1, 96-100.

\section{Note}

1. This work was supported by a grant from the National Aeronautics and Space Administration (Grant R-129) through the U. $S$. Navy Office of Naval Research Contract Nonr-3579(04) and the Department of the Navy, Naval Ship Systems Command Contract NObsr-93124. I thank J. Fisher, M. Reveley, and D. Dydek for their assistance.

(Accepted for publication March 10, 1967.) 